\title{
Chaos and topological entropy in dimension $n>1$
}

\author{
COVADONGA BLANCO GARCÍA \\ Departamento de Ecuaciones Funcionales, Faculdad de Matemáticas, \\ Universidad de Santiago de Compostela (La Coruña), Spain
}

(Received 8 January 1985 and revised 1 July 1985)

\begin{abstract}
In this paper we present a generalization to higher dimensions of the techniques for computation of the entropy of graphs in dimension one. Following these methods, we obtain a lower bound for the topological entropy of a differentiable map $F: \mathbb{R}^{n} \rightarrow \mathbb{R}^{n}$ possessing a snap-back repeller.
\end{abstract}

\section{Introduction}

The mathematical modelling of several real world dynamics problems leads to retarded functional differential equations. If one wishes to obtain numerical results, one is led to discrete difference equations. Thus the evolution (in time) of the process is translated into an iteration of a map $F: I \subset \mathbb{R} \rightarrow \mathbb{R}$, where $I$ is an interval. For this map, one can study, from the point of view of dynamical systems, for instance, the existence of periodic orbits. These orbits are then interpreted, in the model, as real pathological effects. This is usually the case in some physiological phenomenon (haematopoiesis, Cheyne-Stokes breathing, [6], [8]). In some other cases the existence of more complicated behaviours such as the existence of an infinite number of periodic orbits with a random distribution on the domain $I$ of the continuous function $F: I \rightarrow I$ may be more significant. This randomness has been called chaos by $\mathrm{Li}$ and Yorke under the assumption that the following conditions hold:

(H1) For every $k=1,2 \ldots$ there exists a periodic point in $I$ with period $k$.

(H2) There exists a non-denumerable set $S \subset I$ (consisting of non-periodic points) satisfying the following assumptions:

(A) For every $p, q \in S, p \neq q$,

$$
\begin{gathered}
\lim _{n \rightarrow \infty} \sup \left\|F^{n}(p)-F^{n}(q)\right\|>0, \\
\lim _{n \rightarrow \infty} \inf \left\|F^{n}(p)-F^{n}(q)\right\|=0 .
\end{gathered}
$$

(B) For every $p \in S$ and every periodic point $q \in I$

$$
\lim _{n \rightarrow \infty} \sup \left\|F^{n}(p)-F^{n}(q)\right\|>0 \text {. }
$$

We require $F$ to satisfy several conditions in order to guarantee the existence of chaos [5], [4]. A generalization of chaos in the sense of $\mathrm{Li}$ and Yorke, is given in [7] for a differentiable map $F: \mathbb{R}^{n} \rightarrow \mathbb{R}^{n}$. The existence of chaos in this case is assured by the existence of a snap-back repeller for $F$, that is, by the existence of an expansive 
fixed point $Z$ of $F$ in $B_{r}(Z)$ for some $r>0$, such that there exists $X_{0} \in B_{r}(Z)$ with $X_{0} \neq Z, F^{M}\left(X_{0}\right)=Z$ and $\left|D F^{M}\left(X_{0}\right)\right| \neq 0$ for some positive integer $M .\left(B_{r}(X)\right.$ stands for the closed ball in $\mathbb{R}^{n}$ with radius $r$ and centre at $X$, and $\dot{B}_{r}(X)$ its interior.)

However, the information we may have about a system and in consequence about the grade of disorder of it, is understood perfectly when we use the concept of topological entropy (see [1] and especially [3] to compute the topological entropy using the concept of the graph of a map). For this reason, we prefer to characterize the chaos in terms of 'positive topological entropy'. In this direction we have developed a method in [2] where we show the positivity of the topological entropy for a differential equation with delay. In what follows we shall use similar ideas in order to prove a theorem analogous to that of [7] in a more general context.

THEOREM 1. Let $F: \mathbb{R}^{n} \rightarrow \mathbb{R}^{n}$ be a differentiable map. If $F$ has a snap-back repeller, then $F$ has positive topological entropy.

\section{Techniques of entropy of graphs; extension to dimension $n>1$}

The methods of determining entropy of graphs for continuous maps $F: I \rightarrow \mathbb{R}$ or $F: S^{1} \rightarrow S^{1}\left(S^{1}=\{z \in \mathbb{C}:|z|=1\}, I\right.$ a real interval), have been extensively studied in [3]. In what follows we extend the ideas to dimension $n>1$.

Let $F: E \rightarrow E$ continuous, where $E$ is a compact subset of $\mathbb{R}^{n}$. Let $U=\left\{U_{j}, \ldots, U_{s}\right\}$ be an open cover of $E$ such that $U_{i} \cap U_{j}=\varnothing$ for $i \neq j$. Now, let $\bar{U}=\left\{\bar{U}_{1}, \ldots, \bar{U}_{s}\right\}$. Given $K, I \subset E$ compact sets, we say that $I$ is an $F$-cover of $K$ if there exists a compact set $I_{0} \subset I$ such that $F\left(I_{0}\right)=K$. We say that $I F$-covers $K, n$ times if there exist $I_{1}, \ldots, I_{n}$ compact sets with disjoint interiors such that $F\left(I_{i}\right)=K$ for $i=$ $1, \ldots, n$.

Definition 1. A $\bar{U}$-graph of $F$ is a generalized oriented graph with vertices $\bar{U}_{1}, \ldots, \bar{U}_{s}$, such that if $\bar{U}_{i}$ is an $F$-cover of $\bar{U}_{j} n$ times, but not $n+1$ times, then there are $n$, but not $n+1$, arrows from $\bar{U}_{i}$ to $\bar{U}_{j}$.

Using these definitions, we are in a position to construct the matrix associated to the graph and compute its topological entropy following the lines of [3].

\section{Proof of theorem 1; construction of the graph and conclusions}

The hypothesis that $F$ has a snap-back repeller assures the existence of a sequence of compact sets $\left\{B_{k}\right\}_{k=-\infty}^{M}$ (homeomorphic to the open unit ball in $\mathbb{R}^{n}$ ) satisfying:

(a) $B_{k} \rightarrow z$ as $k \rightarrow-\infty$;

(b) $F\left(B_{k}\right)=B_{k+1}$;

(c) $F$ is one-to-one in $B_{k}$;

(d) $B_{k} \cap B_{M}=\varnothing, \quad 1 \leq k \leq M$;

(e) $Z \in \check{B}_{M}$.

In what follows and using this sequence, we shall construct the graph $G$ associated to the map $F$ with respect to itself.

Let $Z$ be a snap-back repeller of $F$, and $X_{0} \in B_{r}(Z), X_{0} \neq Z, F^{M}\left(X_{0}\right)=Z$, and $\left|D F^{M}\left(X_{0}\right)\right| \neq 0$, where $Z$ is expanding in $B_{r}(Z)$. There exists $\varepsilon \in(0, r)$, and a function $G$ defined on $B_{\varepsilon}(Z)$ such that $G$ is one-to-one, $G(Z)=X_{0}, G^{-1}(X)=F^{M}(X)$ for every $X \in G\left(B_{\varepsilon}(Z)\right)$. 
Let $A=G\left[B_{\varepsilon}(Z)\right]$. We consider $B_{0}=A$ and $B_{M}=B_{\varepsilon}(Z)$. Taking into account that $F^{-1}$ is contractive and that $A$ is compact, one can construct the sequence $\left\{B_{k}\right\}_{k=-T}^{M-1},[7]$. Note that if $Z \in B_{-T}$, then there exists $p$ such that $Z \in\left[F^{-p}\left(\ddot{B}_{-T}\right)\right]=$ $\dot{B}_{-T-p}$ and $B_{-T-p}$ satisfies the same conditions as $B_{-T}$ when $Z \notin B_{-T}$.

In the study of the map $F: \mathbb{R}^{n} \rightarrow \mathbb{R}^{n}$, we can restrict to a compact subset of $\mathbb{R}^{n}$ provided that the snap-back repeller belongs to that set. Let $E$ be a compact subset of $\mathbb{R}^{n}$ and assume that $F: E \rightarrow E$. Let $\bar{U}=\left\{B_{k}\right\}_{k=-(T+p)}^{M-1}$ the family of compact subsets we have just constructed. Without loss of generality we can assume that the family of open sets $U=\left\{\check{B}_{k}\right\}_{k=-(T+p)}^{M-1}$ is such that $B_{i} \cap B_{j}=\varnothing$ if $i \neq j$. Using the results of $\S 2$, we can construct the $\bar{U}$-graph, $G$, associated to $F$. If we order the vertices of this graph according to the sequence:

$$
B_{M-1}, B_{-(T+p)} \ldots B_{-T}, B_{-T+1} \ldots B_{-1}, B_{0}, \ldots, B_{M-2},
$$

we get the following matrix $D$ associated to the graph $G$

$$
D=\left[\begin{array}{cccccccccc}
0 & 1 & 1 & \ldots & \ldots & \ldots & 1 & 0 & \ldots & 0 \\
0 & 0 & 1 & 0 & \ldots & \ldots & \ldots & \ldots & \ldots & \ldots \\
0 & 0 & 0 & 1 & 0 & \ldots & \ldots & \ldots & \ldots & 0 \\
\ldots & \ldots & \ldots & \ldots & \ldots & \ldots & \ldots & \ldots & \ldots & \ldots \\
1 & 0 & \ldots & \ldots & \ldots & \ldots & \ldots & \ldots & \ldots & 0
\end{array}\right]
$$

Now, the entropy of the graph $G$ is given by the spectral radius of $D$. In our case, $B_{M-1}$ is a rome. Hence, the characteristic polynomial of $D$ is given by:

$$
p(X)=(-1)^{(p+T+M)-1} X^{(p+T+M)} \phi(X),
$$

where

$$
\phi(X)=X^{-(p+T+M)}+X^{-(p+T+M-1)}+\cdots+X^{-(T+M)}-1 .
$$

Studying the zeros of this polynomial, we can write $h(G)>0$. Finally, by [3] we know that

$$
h(F) \geq h(G) \text {. }
$$

This gives us a positive lower bound for the topological entropy of the map $F$.

\section{REFERENCES}

[1] R. L. Adler, A. G. Konheim \& M. H. McAndrew. Topological entropy. Trans. Amer. Math. Soc. 114 (1965), 309-319.

[2] C. Blanco \& J. A. Rodríguez. Caos y entropía topológica para la ecuación diferencial con retardo. Actas VI C.E.D.Y.A. pp. 220-226 Zaragoza (1983).

[3] L. Block, J. Guckenheimer, M. Misiurewicz \& L.-S. Young. Periodic points and topological entropy of one dimensional maps. In Global Theory of Dynamical Systems, Proceedings, pp. 18-34. NorthWestern, 1979. Lecture Notes in Math. No 819. Springer: Berlin, 1980.

[4] U. Heiden \& H.O. Walther. Existence of chaos in control systems with delayed feedback. $J$. Differential Equations. 47. No 2 (1983), 1-29.

[5] T.-Y. Li \& J. A. Yorke. Period three implies chaos. Amer. Math. Monthly. 82 (1975), 985-992.

[6] M. Mackey \& L. Glass. Oscillations and chaos in physiological control systems. Science 197 (1977), 287-299.

[7] F. R. Marotto. Snap-back repellers imply chaos in $\mathbf{R}^{n}$. J. Math. Anal. \& Appl. 63 (1978), 199-223.

[8] H. C. Morris, E. E. Ryan \& R. K. Dodd. Periodic solutions and chaos in a delay-differential equation modelling haematopoiesis. Nonlinear Anal. 7. No 6 (1983), 523-660. 